\title{
The Multiobjective Based Large-Scale Electric Vehicle Charging Behaviours Analysis
}

\author{
Yimin Zhou $\mathbb{D}^{1,2}$ Zhifei Li, ${ }^{1,2}$ and Xinyu Wu $\mathbb{D}^{1,3}$ \\ ${ }^{1}$ Shenzhen Institutes of Advanced Technology, Chinese Academy of Sciences, Shenzhen, China \\ ${ }^{2}$ Guangdong Provincial Engineering Technology Research Center of Intelligent Unmanned System and Autonomous \\ Environmental Perception, 518055, China \\ ${ }^{3}$ Guangdong Provincial Key Lab of Robotics and Intelligent System, Shenzhen Institutes of Advanced Technology, \\ Shenzhen 518055, China \\ Correspondence should be addressed to Xinyu Wu; xy.wu@siat.ac.cn
}

Received 27 March 2018; Revised 7 August 2018; Accepted 4 September 2018; Published 16 October 2018

Academic Editor: Chongyang Liu

Copyright (c) 2018 Yimin Zhou et al. This is an open access article distributed under the Creative Commons Attribution License, which permits unrestricted use, distribution, and reproduction in any medium, provided the original work is properly cited.

\begin{abstract}
In the paper, the effect of the charging behaviours of electric vehicles (EVs) on the grid load is discussed. The residential traveling historical data of EVs are analyzed and fitted to predict their probability distribution, so that the models of the traveling patterns can be established. A nonlinear stochastic programming model with the maximized comprehensive index is developed to analyze the charging schemes, and a heuristic searching algorithm is used for the optimal parameters configuration. With the comparison of the evaluation criteria, the multiobjective strategy is more appropriate than the single-objective strategy for the charging, i.e., electricity price. Furthermore, considering the characteristics of the normal batteries and charging piles, user behaviour and EV scale, a Monte Carlo simulation process is designed to simulate the large-scale EVs traveling behaviours in long-term periods. The obtained simulation results can provide prediction for the analysis of the energy demand growth tendency of the future EVs regulation. As a precedent of open-source simulation system, this paper provides a stand-alone strategy and architecture to regulate the EV charging behaviours without the unified monitoring or management of the grid.
\end{abstract}

\section{Introduction}

With the gradually deteriorated air quality, environments and energy crisis caused by the fuel-powered vehicles around the world, renewable vehicles, i.e., electric vehicles (EVs) are greatly promoted by all the governments, and many policies have been issued related to their development, where EVs will become the main transportation tools in the future along with the increasing improved technologies and infrastructure construction. However, the continuous energy demand growth of EVs puts a heavy pressure on the power grid with serious uncertainties in grid regulation [1]. Generally, one $\mathrm{EV}$ connection to the grid is roughly equal to the load requirement from one small household, where the construction of the grid in California, the most popular states of EVs in America, is facing the grid update problem currently [2]. The effect of EVs on the distributed power grid has been investigated in [3-5], and the involved impact factors can be summarized as traveling patterns, battery characteristics, charging schedule, and EV penetration, as shown in Figure 1.

From the analysis of the Danish national transportation survey data, the EV traveling model can be established with the driving distance and driving periods as the statistical data, so that the power demand and expected charging time from EVs can be determined [6]. The data demonstrate that the average driving distance of the EVs is $29.48 \mathrm{~km}$, while the daily driving distance of $75 \%$ of the EVs is less than $40 \mathrm{~km}$. Therefore, $40 \mathrm{~km}$ driving distance can be used to determine whether the EV battery capacity can meet the daily traveling requirement. Moreover, EVs can be considered as the mobile load connected between the power system and the transportation system, where the random mobility and charging of the EVs are dependent on the stochastic traveling patterns 


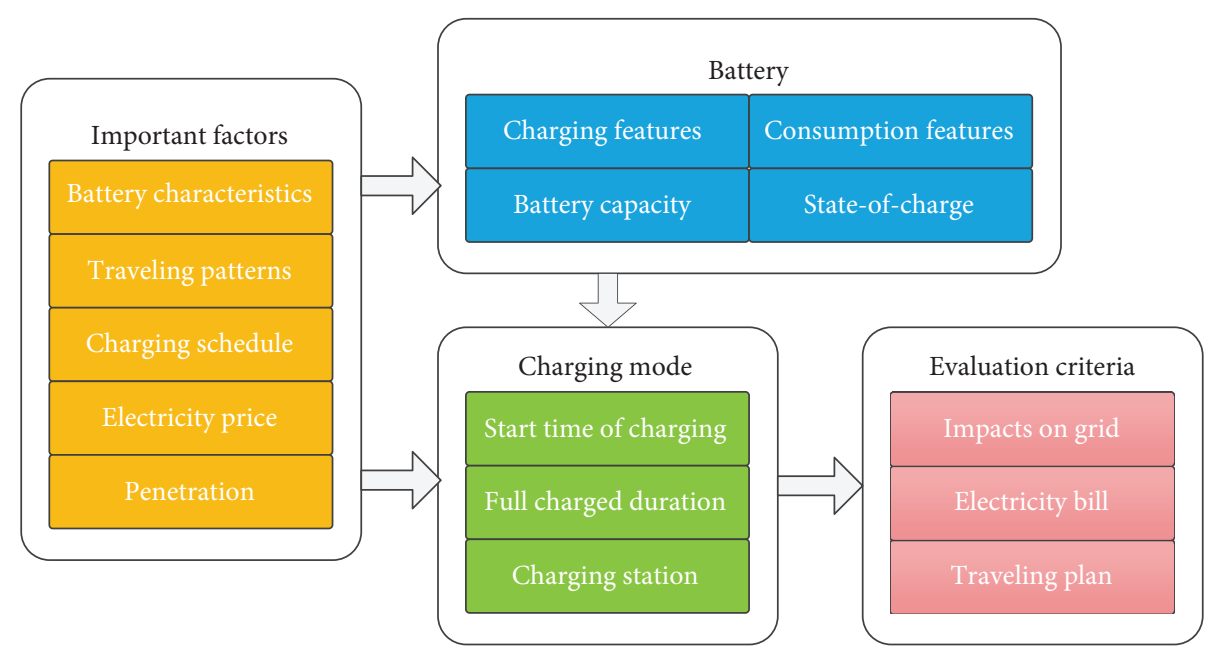

FIGURE 1: The impact factors of EV charging to the power system.

of the EV owners [7]. The graph theory was used to analyze the traffic and power network based on the driving patterns, and the randomly mobilized characteristics of the EV charging load was modelled accordingly.

Besides the requirement of the power quality (i.e., voltage and frequency) during EV charging, the required charging power is the most concerned for the power supply utilities [8]. According to the standard J1772 of the Society of Automotive Engineers [9], charging power can be divided into three levels, where grade $1(1.5-3 \mathrm{~kW})$ and grade 2 $(10-20 \mathrm{~kW})$ are suitable for family use, and grade $3(40 \mathrm{~kW}$ and above) is more suitable for EV fast charging, by which EV charging can be completed within one hour; however, it is mostly used in centralized charging scenarios due to overly high power.

The charging plan is normally determined by two factors, charging availability (charging situation) and charging strategy [10]. Since the energy consumption of the daily EV journey is less than the battery-rated capacity, it is unnecessary to charge the EV on daily basis. The EV can start to be charged only when its battery state-ofcharge (SOC) is less than the threshold. The EV charging strategies play an important role in the evaluation of the $\mathrm{EV}$ influence on the power system [11], which can be divided into (1) simple charging (dumb charging), i.e., the unplanned "plug and play" pattern, generally charged at the end of the trip in one day or when the charging facility is available; (2) tariff-driven charging, i.e., charging during off-peak periods with cheaper cost; and (3) intelligent charging, i.e., the extra battery energy can be used as the energy resource to provide assistant service during high-peak hours, which is beneficial to the stable grid operation.

Another important aspect to analyze the EVs influence on the grid is to design the evaluation index quantitatively. In [12], based on the insular power system of Crete in Greece, it studied the impact of plug-in EVs with smart and direct charging patterns on the grid energy scheduling and cost. An EV aggregator that participates in the market is proposed with the price-taking approach for solving optimally self-scheduling problem. Compared with different scenarios of EV penetration, an irregular performance would be presented on the unit commitment schedule while the number of EVs reaches 21,000. Paper [13] introduces an architecture for the intelligent energy management system, which is composed of an admission control, a pricing module, and a power scheduling module that determines the charging sequence of EVs. The queuing problem of power dispatch is formulated as a stochastic dynamic programming problem, and a threshold admission and greedy scheduling method is adopted to minimize the electricity cost.

Based on the review of EVs impacts on the grid, it can be shown that EVs popularization will bring nonneglectful new electric load. At present, however, most research conclusions about large-scale EVs charging are drawn from simulation experiments. There are obvious discrepancies of gas station facilities and EV supporting facilities among different cities. How to improve the credibility and universality of the prediction results is still a challenge. Therefore, many involved issues should be addressed to improve the load prediction accuracy, i.e., driving and charging models with appropriately practical condition, traveling probability distribution model, and multiobjective EV charging patterns.

Furthermore, most works merely consider the price incentive in the charging modes, and the relation between EVs traveling behaviours and charging schemes has not been accurately described by mathematical models $[14,15]$. This paper investigates large-scale EV charging behaviours as a multiobjective optimization problem, and the main contributions are summarized as follows:

(1) From the benefits of the EV users and power suppliers perspectives, the comprehensive evaluation index system has been developed with three key factors: load peak value, charging bills, and traveling rate, which are used as the objective to describe the 


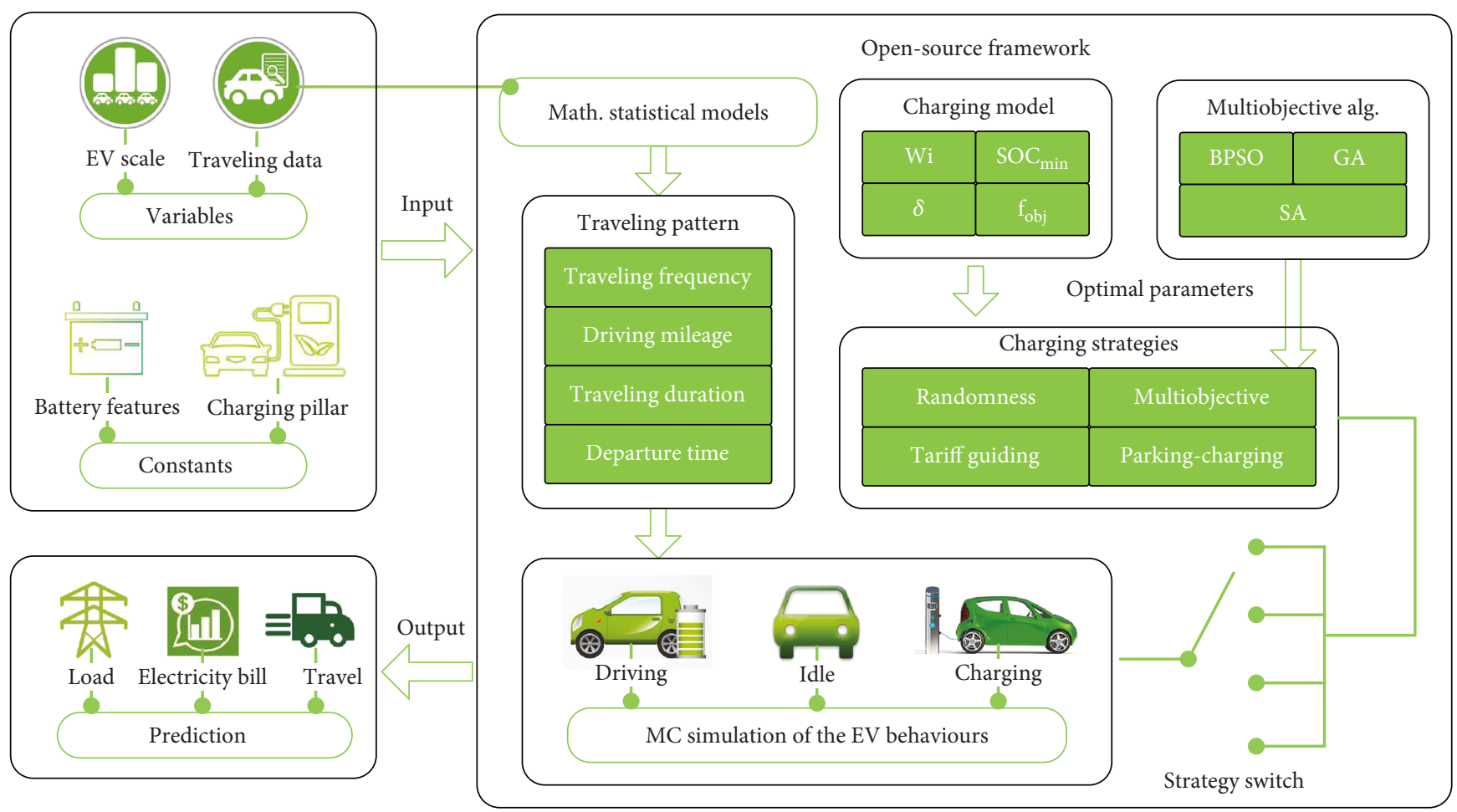

FIGURE 2: The framework for EVs traveling and charging behaviours analysis.

prediction and optimization problems of the EVs power usage requirement

(2) Based on the actual traffic data, the probability distribution model of the traveling patterns is established; Monte Carlo (MC) is adopted to establish the largescale EVs traveling and charging simulation system to improve the load prediction accuracy

(3) A multiobjective charging strategy has been developed under multiple constraints, and the involved parameters are determined via a novel constraint mechanism which transfers the equality-boundary constraints into binary feature selection

(4) Besides, the nonlinear model of the charging behaviour and the MC simulation algorithm have been fully open-source [16]. Based on the proposed framework shown in Figure 2, more grid-impact assessments can be focused on the situation of EVs in different districts and their charging facilities

The remainder of the paper is organized as follows. Section 2 analyzes the energy usage status of the EVs, and the optimized indexes are determined. The statistical model of the traveling pattern and the charging strategies of the EVs are introduced in Section 3. Monte Carlo simulation algorithm is used to calculate the solution of the nonlinear programming model in the same section. In Section 4, a multiobjective charging strategy is developed with optimal parameters, and the proposed strategies are compared via simulation experiments as well. Conclusions and future work are given in Section 5.

\section{Problem Formulation}

2.1. System Description. According to the traffic survey in Beijing [17], the traveling periods of private EVs during working days are concentrated among 6:00-9:00 (early peak) and 16:00-19:00 (evening peak), and $40.3 \%$ of the parking time (slack time $>5$ hours) of EVs are distributed among 18:00-21:00, which are depicted in Figure 3. If EVs charging is performed without any guidance, peak load would be generated due to the large-scale charging concentrated at parking periods. According to the 2020 Shenzhen EVs growth plan [18], the current power grid will be difficult to satisfy the energy demand of EVs, which would result in power supply tension, or even endanger the safety of the grid operation [19]. Furthermore, more investment should be put to satisfy higher load peak requirement, resulting in large-scaled equipment upgrade and capital budget pressure. On the other hand, due to the constrained battery capacity and limited charging piles, the EVs traveling plan would be hindered if without appropriate charging schedule, which is currently one of the most important reasons to limit the popularity of EVs.

Therefore, an objective to study the effect of large-scaled $\mathrm{EV}$ charging on the grid load (hereinafter referred to as "EV charging problem") is summarized as: based on the routine traveling habits of EV users, the optimal charging strategy is proposed to reduce the grid peak load, lower the cost of EV users, and guarantee the success of traveling plan, which can be ascribed as one of the NP-hard problems.

First, the relationship between the traveling patterns and charging behaviour are analyzed so as to extract the involved 


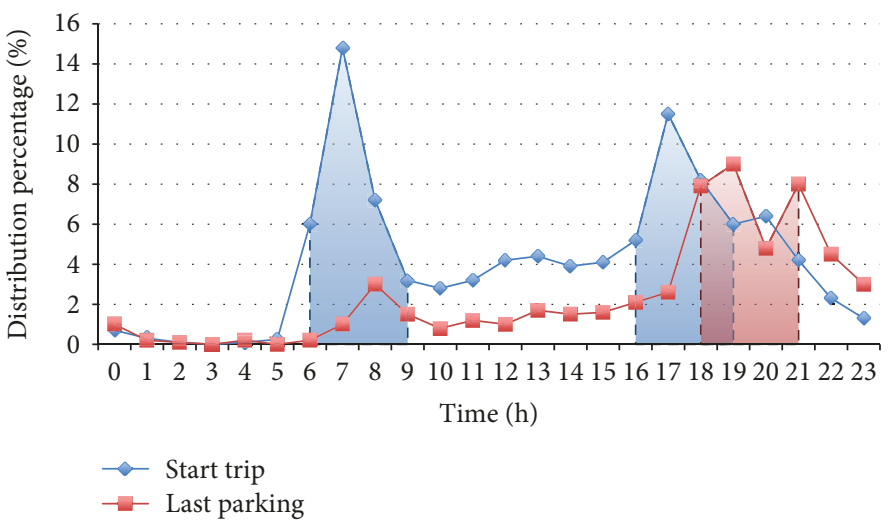

FIGURE 3: Daily traveling and parking behaviours of EVs in Beijing.

key factors and determine the evaluation indices. The total required power of the $\mathrm{EV}$ charging from the grid system at the specific moment is determined by the four main impact factors, i.e., battery characteristics (Cap $\sim \mathrm{SOC})$, charging piles $\left(W_{c}\right)$, users behaviour $\left(F_{d} \sim Q_{c}\right)$, and the current EV number $\left(N_{\mathrm{ev}}\right)$. As it is shown, the characteristics of the battery pack, charging piles, and current EV number can be assumed as known variables. The variables describing user behaviours are unknown variables which will be determined by mathematical models.

For this type of NP-hard problems which cannot be solved directly, the MC simulation method is adopted to simulate the EVs operation system under different charging strategies for the purpose of optimal EV charging mode obtainment. Based on the statistics, different probability distributions are selected to describe the EVs driving behaviours to improve the credibility of the MC simulation results. Finally, several commonly used optimization algorithms are introduced to adjust the parameters configuration of the charging modes so as to realize the optimal comprehensive index.

According to the technical specifications of the small EVs and common characteristics of urban residents' vehicle traveling habit, several assumptions have been made to describe the complex EVs charging problem without loss of generality.

(1) The EV battery and parameters related to the charging piles are set similar to those of the commercial products

(2) Each day (24 hours) will be divided equally into 96 intervals, i.e., 15 minutes as the sampling period

(3) The traveling frequency, departure-parking time, and driving mileage are the main factors directly affecting the $\mathrm{EV}$ charging behaviour, i.e., the charging period $\left(T_{c}\right)$, initial SOC $\left(\mathrm{SOC}_{\mathrm{ini}}\right)$, and the required charging quantity $\left(Q_{c}\right)$. So the traveling patterns and the charging strategies are the two key factors to be focused

(4) In order to guarantee the success of the expected traveling plan, it is assumed that the battery packs will be fully charged at each charging
(5) The traveling patterns of the personal EVs on the working days are considered; other types of EVs or driving patterns under holidays/weekends will not be addressed unless otherwise specified

(6) The urban residents in Shenzhen and in Beijing share the same traveling pattern

Replacing the input module (shown in Figure 2) with specific traffic data, the problem formulation and models can be extended to other urban areas or holiday/weekend driving patterns. Thus, the designed framework has the advantage of solving a universal problem and benefits to obtain reasonable results in different situations.

2.2. The Evaluation Index System. The different evaluation indices are listed in Nomenclature after Conclusions, which are developed from the perspectives of power suppliers $\left\{W_{\text {peak }}, W_{\text {average }}, \mathrm{APR}, R_{\text {load }}\right\}$ and EV owners $\left\{C, C_{\max }\right.$, $\left.R_{\text {save }}, R_{\text {trip }}\right\}$. Since the average-peak ratio (APR), saving rate $\left(R_{\text {save }}\right)$, and traveling rate $\left(R_{\text {trip }}\right)$ are the most concerned indices for the energy suppliers and EV owners [10,20], they are used to form the comprehensive index $Y$, which is defined as

$$
\left\{\begin{array}{c}
Y=\alpha \cdot\|\mathrm{APR}\|+\beta \cdot\left\|R_{\text {save }}\right\|+\gamma \cdot\left\|R_{\text {trip }}\right\|, \\
1.0=\alpha+\beta+\gamma,
\end{array}\right.
$$

$$
\|x\|=\frac{\left(x-X_{\min }\right)}{\left(X_{\max }-X_{\min }\right)}
$$

where $\alpha, \beta$, and $\gamma$ are the positive weight coefficients $(\leq 1.0)$, and they can be set flexibly. Here, $\alpha=0.4, \beta=0.3$, and $\gamma=0.3$, denoting load balance index taken as priority. The higher value of $Y$ means higher system performance. $\|\cdot\|$ is used to normalize the index value by equation (2), and $\left[X_{\min }, X_{\max }\right]$ are bound of $X$. The normalization can improve the sensitivity of the optimization algorithm to the index variation so as to avoid premature convergence. 
TABLE 1: The involved five factors for EV traveling pattern analysis.

\begin{tabular}{lcccc}
\hline Total distance & Distance per trip & Travel duration & Duration per trip & Travel times \\
\hline $35.4 \mathrm{~km}$ & $15.5 \mathrm{~km}$ & $1.51 \mathrm{~h}$ & $0.63 \mathrm{~h}$ & 2.29 \\
\hline
\end{tabular}

\section{The Ev Load Estimation Based on Monte Carlo Simulation}

After the optimal objective and key impact factors are determined, the EV charging problem can be configured as a nonlinear programming model. Under the constraints of the EV battery characteristics and user traveling patterns, the objective functions can be established based on different demands from the power suppliers and EV users so as to obtain the optimal charging schemes. The undetermined variables in the model, i.e., $W_{i}$, are quantified via $\mathrm{MC}$ simulation tool to simulate the daily EV traveling, charging, and slack state. Furthermore, the statistic models of the traveling habit and EV charging behaviour have been established to improve the MC simulation credibility level.

3.1. The Probability Distributed Fitting Model for the Traveling Variables. According to the data collected from the GPS installed on 112 private EVs in Beijing [17], totally, 4892 data from June 2012 to March 2013 are recorded and used as the relevant information are outlined in Table 1 . Here, from the analysis of the EVs driving data in working days, the probabilistic density function (PDF) and its parameters of the traveling variables can be determined as follows (see Figure 4):

(1) To select the PDF from the generally used probabilistic functions, i.e., exponential, gamma, normal, and Poisson distribution functions, which are called "PDF-x"

(2) To estimate the scale and shape parameters of the "PDF-x" through the maximum likelihood estimation (MLE) [21]

(3) To verify whether the generated 1000 groups of data accept the original hypothesis at a confidence level of 95\% (significance level $\alpha=0.05$ ) by the K-S, F-test, and $T$-test such statistical methods [22]

(4) According to the $P$ value results, the fitting degree between the "PDF-x" model is obtained

(5) To evaluate the best fitting of the "PDF" as "PDFbest" after iterative comparison

Then the obtained "PDF-best" of each variable is applied to the simulated system so the input values satisfied specific probabilistic distributions are in accordance with the actual vehicles traveling patterns. Although there are certain biases existence at $F_{d} D_{t}^{\mathrm{am}}$ and $D_{t}^{\mathrm{pm}}$ (shown as Figures 5(a) and 5(d)), different hypotheses testing methods (including chi-square test, Kolmogorov-Smirnov test, and $t$-test) are adopted and verified that these deviations are acceptable with sufficient statistical samples, the detailed results are achievable in our open-source project [16]. Therefore, the designed MC simulation model is quite reliable with high credibility.

(1) The daily traveling frequency, $F_{d}$, follows $\Gamma$ distribution,

$$
\begin{aligned}
f(x \mid a, b) & =\frac{1}{b^{a} \Gamma(a)} x^{a-1} e^{(-x / b)}, \\
\Gamma(a) & =\int_{0}^{\infty} t^{a-1} e^{-t} d t,
\end{aligned}
$$

where $\Gamma(a)$ is the gamma function, $x$ is the random variable, $f(\cdot)$ is the PDF of $F_{d}$, and $a$ and $b$ are the shape parameter and scale parameter, respectively. Through the MLE, $a=3.71, b=0.64$, the expectation mean $=2.39$, and the variance var $=$ 1.24 can be obtained. The comparison between the actual data and the fitted curve of the traveling frequency is shown in Figure 5(a)

(2) The driving mileage of each traveling, $M_{d}$, follows Birnbaum-Saunders (BS) distribution,

$$
\begin{aligned}
f(x \mid \beta, \gamma)= & \frac{1}{\sqrt{2 \pi}} \cdot\left\{\exp \left[-\frac{(\sqrt{x / \beta}-\sqrt{\beta / x})^{2}}{2 \gamma^{2}}\right]\right\} \\
& \cdot \frac{(\sqrt{x / \beta}+\sqrt{\beta / x})}{2 \gamma x},
\end{aligned}
$$

where $f(\cdot)$ is the PDF of the BS distribution; $\beta$ and $\gamma$ are the scale parameter and shape parameter, respectively. Through the MLE, $\beta=10.57, \gamma=$ 0.97 , mean $=15.52$, and var $=15.09$ can be obtained. The probability density distribution curves of the actual data and the BS fitting curve are shown in Figure 5(b)

(3) The duration of each traveling, $T_{d}$, follows $\Gamma$ distribution. Suppose each traveling duration also obeys gamma distribution as expressed in equation (3), the parameters can be acquired via MLE, i.e., $a=$ $1.87, b=18.35$, mean $=34.4$, and $\operatorname{var}=25.12$. The fitted curve with gamma distribution and the actual data curve are depicted in Figure 5(c). 


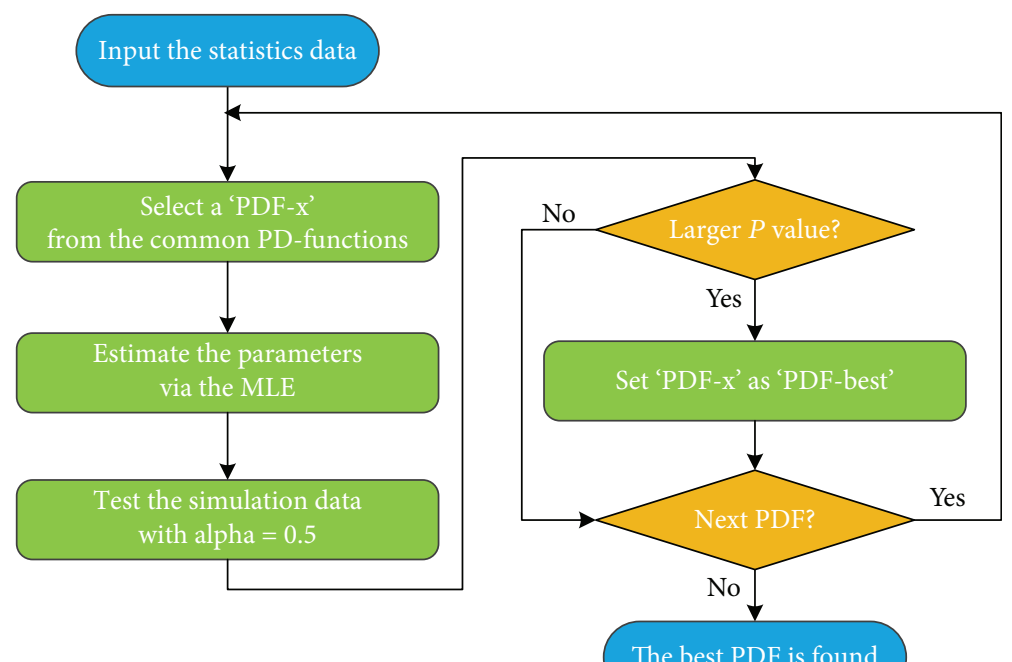

The best PDF is found

FIgURE 4: Diagram of the distribution function selection of the involved traveling variables.

(4a) The departure time of each traveling (AM), $D_{t}^{\mathrm{am}}$, follows a location-scale distribution,

$$
\begin{aligned}
f_{\mathrm{D}_{t}^{\mathrm{am}}}(x \mid \mu, \sigma, v)= & \frac{\Gamma((v+1) / 2)}{\sigma \cdot \sqrt{v \pi} \cdot \Gamma(v / 2)} \\
& \cdot\left[\frac{v+((x-\mu) / \sigma)^{2}}{v}\right]^{-((v+1) / 2)},
\end{aligned}
$$

where $f(\cdot)$ is the $\mathrm{PDF}$ of $D_{t}^{\mathrm{am}}, \Gamma(\cdot)$ is the gamma function, and $\mu, \sigma, \nu$ are the location parameter, scale parameter, and shape parameter, respectively. Through the MLE, mean = $\mu=8.36, \quad \sigma=1.08, \quad v=2.16$, and $\operatorname{var}=3.98$ can be obtained.

(4b) The departure time of each traveling $(\mathrm{PM}), D_{t}^{\mathrm{pm}}$, follows normal distribution,

$$
f_{D_{t}^{\mathrm{pm}}}(x \mid \mu, \sigma)=\frac{1}{\sigma \sqrt{2 \pi}} \cdot e^{\left(-(x-\mu)^{2}\right) /(2 \sigma)^{2}} .
$$

Through the MLE, the expectation $\mu=18.2$ and the standard variance $\sigma=2.84$. The fitted curve with probability density distribution and the actual data curve are shown in Figure 5(d).

3.2. Modelling the Charging Strategies. EV users would select different charging strategies, i.e., to set a specific target or to charge at optimal periods. For instance, under the incentive of the time-of-use (ToU) electricity prices [23], the users would charge EVs during parking periods with lower electricity prices, which is called the tariff-guiding strategy. The usually used three charging strategies are described in Table 2.
Equation (7) describes the unified quantization formula of the users' charging motivation. By changing the weight coefficient $W_{i}(i=1,2,3)$ of the three charging strategies, the charging strategy models can be derived quantitatively as follows:

$$
\left\{\begin{array}{c}
P=W_{1} R+W_{2}\left(1.5-\frac{\bar{C}}{\bar{C}_{\text {TOC }}}\right)+W_{3}\left(1.5-\frac{T_{\text {charge }}}{T_{\text {slack }}}\right)+U(s), \\
1.0=\sum_{i=1}^{3} W_{i}, \quad W_{i} \in[0.0,1.0],
\end{array}\right.
$$

$U(s)=\left\{\begin{array}{c}+\infty, \mathrm{SOC}_{\text {curr }} \leq \mathrm{SOC}_{\min } \\ 0, \text { otherwise }\end{array}\right.$

where $P$ is the charging priority to be arranged at certain period, i.e., EV starts charging when $P>0.5 ; R$ is the random priority factor following the uniform distribution with $R \in[0,1]$, in order to simulate the random charging behaviour; $T_{\text {charge }}$ and $T_{\text {slack }}$ are the predicted time intervals required for the fully charged and the slack time intervals, respectively; $U(s)$ denotes that if the current SOC of the $\mathrm{EV}, \mathrm{SOC}_{\text {curr }} \leq \mathrm{SOC}_{\min }$, the EV would be charged immediately. In order to ensure the reliability of the traveling plan, $\mathrm{SOC}_{\min }=0.2 . \bar{C}$ and $\bar{C}_{\mathrm{TOC}}$ are the average charging cost at certain periods and the average charging cost of one day, which can be calculated as follows:

$$
\bar{C}=\frac{\sum_{i=1}^{T} C_{i}}{T},
$$

where $T$ is the continuous charging duration and $C_{i}$ is the ToU price at the $i^{\text {th }}$ moment, as listed in Table 3; here, $\bar{C}_{\text {TOC }}=0.57$ Yuan $/ \mathrm{kWh}$.

Through the comparison of the priority level at each interval during EV slack periods, the charging moment can 


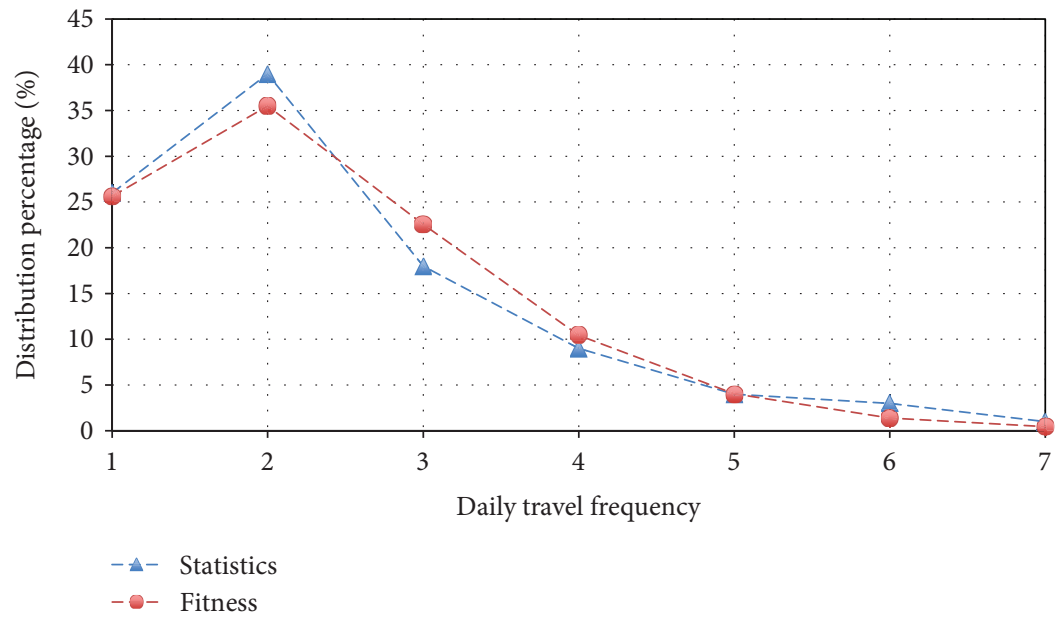

(a) The distribution of the daily traveling frequency $F_{d}$

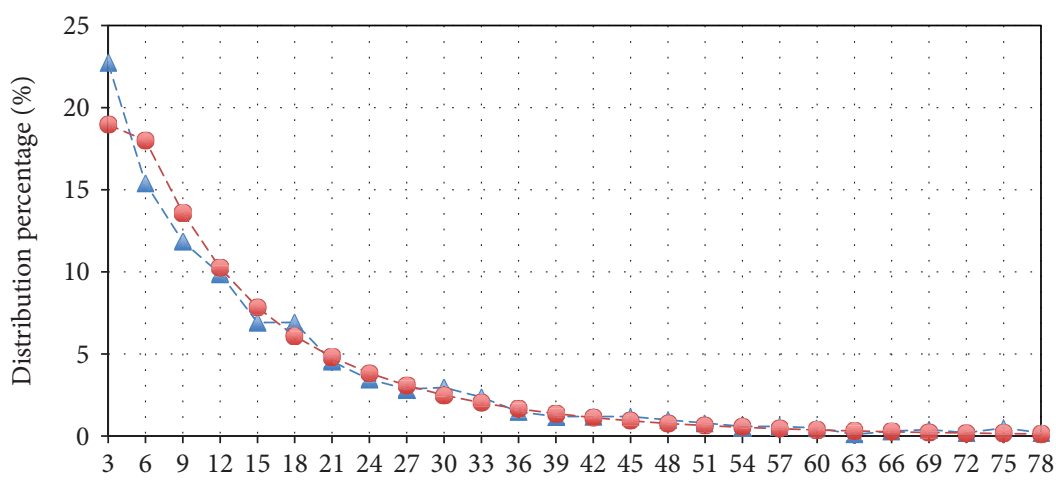

Driving mileage per trip $(\mathrm{km})$

$-\AA-$ Statistics

- - Fitness

(b) The distribution of the driving mileage of each traveling $M_{d}$

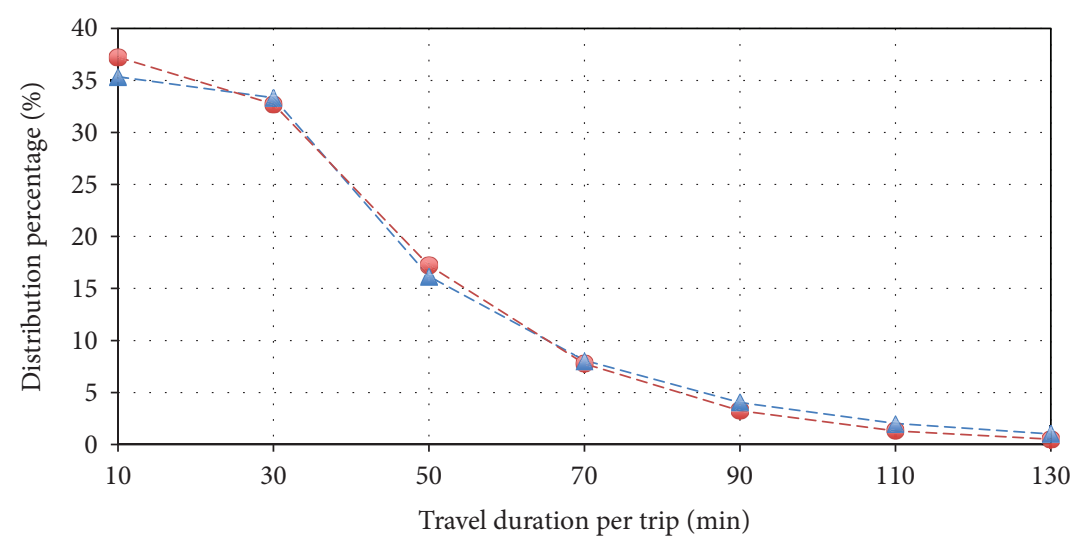

$-\AA-$ Statistics

- Fitness

(c) The distribution of the duration of each traveling $T_{d}$

Figure 5: Continued. 


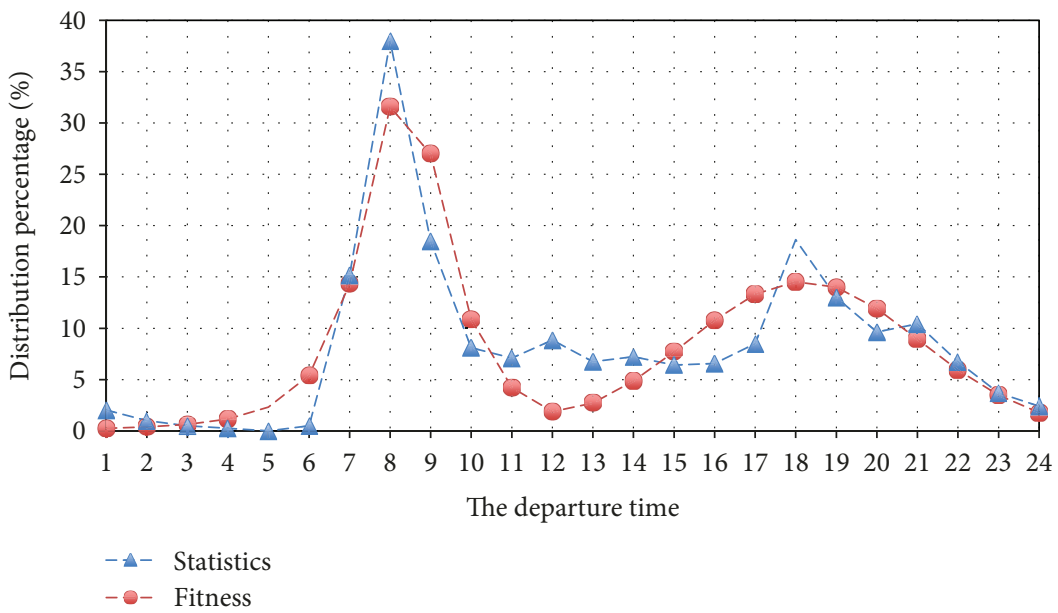

(d) The distribution of the departure time of each traveling (AM/PM) $D_{t}^{\mathrm{am}}$ and $D_{t}^{\mathrm{pm}}$

FIGURE 5: Fitting analysis of the EVs traveling historical data.

TABLE 2: Description of the charging strategies.

\begin{tabular}{|c|c|}
\hline Strategy & Description \\
\hline Randomness & $\begin{array}{l}\text { When } W_{1}=1.0 \text { and } \mathrm{SOC}_{\text {curr }}>\mathrm{SOC}_{\min } \text {, charging at }\{P=R\}>0.5 \text { denotes that charging is a random behaviour that } \\
\text { accords with the uniform distribution. }\end{array}$ \\
\hline Tariff guidance & $\begin{array}{c}\text { When } W_{2}=1.0 \text { and } \mathrm{SOC}_{\text {curr }}>\mathrm{SOC}_{\text {min }} \text {, charging at }\left\{P=\left(1.5-\bar{C} / \bar{C}_{\mathrm{TOC}}\right)\right\}>0.5 \text { denotes that the charging starts when } \\
\text { the current average cost of the charge is lower than that of the daily average cost, i.e., } \bar{C}<\bar{C}_{\mathrm{TOC}} \text {, that encourages users to } \\
\text { charge EVs at the parking period with the lowest electricity prices. }\end{array}$ \\
\hline $\begin{array}{l}\text { Charging at } \\
\text { parking }\end{array}$ & $\begin{array}{l}\text { When } W_{3}=1.0 \text { and } \mathrm{SOC}_{\text {curr }}>\mathrm{SOC}_{\text {min }} \text {, charging at }\left\{P=\left(1.5-T_{\text {charge }} / T_{\text {slack }}\right)\right\}>0.5 \text { denotes that the charging will be } \\
\text { started immediately after the arrival if the parking time is longer than the required full charging time, i.e., } T_{\text {charge }}<T_{\text {slack }} \\
\text { since } T_{\text {slack }} \text { decreases over time, the maximum priority value } P \text { can be achieved at the just parking moment. }\end{array}$ \\
\hline
\end{tabular}

TABle 3: Time-of-use electricity prices per day (unit : Yuan/kWh) [23].

\begin{tabular}{lcccc}
\hline$[0-7] h$ & [7-9]h & {$[\mathbf{9 - 1 1}] \mathbf{h}$} & {$[\mathbf{1 1 - 1 4}] \mathbf{h}$} & {$[\mathbf{1 4 - 1 6}] \mathbf{h}$} \\
0.23 & 0.61 & 0.92 & 0.61 & 0.92 \\
{$[16-19] \mathrm{h}$} & {$[\mathbf{1 9 - 2 1}] \mathbf{h}$} & {$[\mathbf{2 1 - 2 3}] \mathbf{h}$} & {$[\mathbf{2 3 - 2 4}] \mathbf{h}$} & \\
0.61 & 0.92 & 0.61 & 0.23 & \\
\hline
\end{tabular}

be regarded as the highest priority (e.g., the maximum random value, the least cost, or the longest parking time) to achieve the maximum benefit. The pseudocode of the charging procedure is shown in Algorithm 1.

3.3. Monte Carlo Simulation. Based on the EV traveling and charging statistics, the MC is used for numerical simulation to solve the output performance index of the different charging schemes.

3.3.1. The Basic Concept of the MC Method. MC method is known as a stochastic simulation method or statistical testing method to use random sampling for mathematical function estimation. MC has statistical convergence where the fitting deviation converges to a certain threshold.

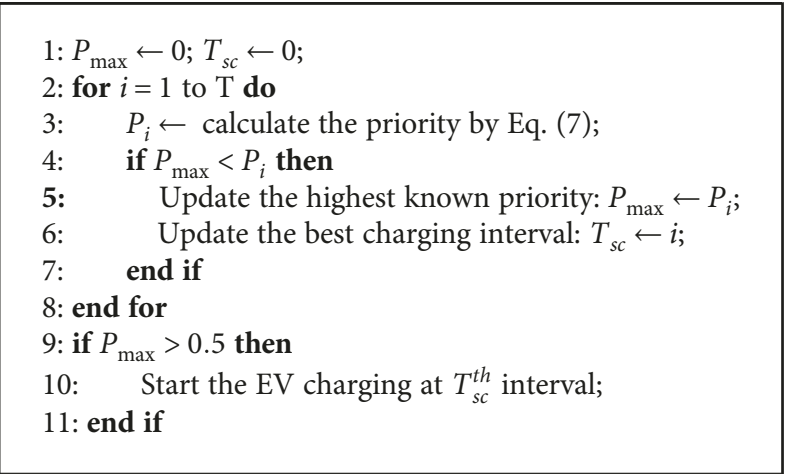

Algorithm 1: The EV charging procedure.

The predicted model of the EV charging capacity calculation is developed based on MC random sampling tests. The capacity of the battery charging is calculated per day evenly divided into 96 intervals, and the total charging capacity in the $i^{\text {th }}$ time interval is described as

$$
H_{i}=\frac{1}{D} \cdot \sum_{d=1}^{D}\left(\sum_{j=1}^{N} h_{i j}(d)\right)
$$


where $h_{i j}(d)$ represents the charging capacity of the $j^{\text {th }} \mathrm{EV}$ in the $i^{\text {th }}$ time slot on the $d^{\text {th }}$ workday, $N$ represents the number of the EVs acquiring power supply from the grid in the $i^{\text {th }}$ time period, and $D$ is the total counted days. Several constraints are set in the simulation. Consider the condition that the battery should be in fully charged status before driving, the starting time of the battery charging should be limited as

$$
\begin{aligned}
\Delta T_{j} & =\frac{Q c_{j}}{W c}=\frac{\left(1-\mathrm{SOC}_{\mathrm{ini}, j}\right) \cdot \mathrm{Cap} \cdot \mathrm{Vol}}{W c}, \\
t_{j} & \in\left(T_{0 j}, T_{1 j}-\Delta T_{j}\right),
\end{aligned}
$$

where $T_{0 j}$ and $T_{1 j}$ are the starting and ending slack status of the $j^{\text {th }} \mathrm{EV}, \Delta T_{j}$ is the maximum continuous charging duration, $Q c_{j}$ is the charging capacity, $\mathrm{SOC}_{\mathrm{ini}, \mathrm{j}}$ is the initial state of the $j^{\text {th }}$ battery capacity that is related to the driving distance per trip, and $t_{j}$ delegates the starting charging moment under fully charged conditions. The condition of convergence adopted in MC sampling model is expressed as

$$
\beta_{i}=\frac{\sqrt{V_{i}(\bar{y})}}{\overline{y_{i}}}=\frac{\sigma_{i}(\bar{y})}{\overline{y_{i}}},
$$

where $\beta_{i}$ is the variance coefficient of the system indicator at the $i^{\text {th }}$ moment; $V_{i}, \overline{y_{i}}$, and $\sigma_{i}$ are the variance, expectation, and standard deviation. The repeated times in MC simulation is at least 100 , and the variance coefficient $\beta_{i}$ is set to less than $0.5 \%$.

3.3.2. The Comprehensive Index Calculation via $M C$ Simulation. Firstly, according to the users traveling model, the daily driving duration and mileage are determined, while the EV charging time period is dependent on the traveling status, characteristics of the battery, and the adopted charging strategy. After the determination of the EV driving/ charging period, the total driving mileage, charging power, and charging bills can be calculated in the 96 time intervals per day. Finally, the comprehensive index and related variance coefficients can be studied based on equations (1) and (12) to complete the whole day simulation.

According to the Shenzhen EVs demonstration and promotion plan, the number of private EVs will reach 240,000 in 2020, which will be used for the charging load calculation. Based on the probability distribution model of the traveling pattern and three types of charging strategies, the simulated large-scale EVs operation procedure via MC simulation is shown in Figure 6. The related indices of the predicted grid load per day in 2020 are shown in Table 4 .

It can be seen from Table 4 that there are large difference for the peak loads or APR with different charging strategies. Compared to the traveling habits of EV users, the EVs charging behaviours are more controllable via certain guidance. Hence, the charging strategy is one of the most feasible optimization objectives.

\section{Multiobjective Charging Strategies for the Large-Scale Evs}

According to the government report, the grid load of the Shenzhen exceeded 15598.6 MW at 11:10 AM of 27th June 2016, and the EV total charging load will exceed $5000 \mathrm{MW}$ per day in 2020. Moreover, with random and parking-charging patterns, EV owners select to perform charging during peak periods, i.e., 9 AM-14 PM and 20-23 PM, which is $1.57 \%$ and $1.97 \%$ of the historical peak value, respectively. From the grid safety perspective, it could easily cause peak superposition of the electricity usages, thus putting heavy pressure on the grid accommodation capacity obviously.

On the other hand, EVs charging with tariff guidance can avoid the charging during high-peak periods but could generate another charging peak value $824.64 \mathrm{MW}$ in shorter periods between 23:00-2:00, which is shown in Figure 7 . Thus, with only the adoption of ToU pricing mechanism, it would result in other concentrated charging periods and abruptly steep high-peak electricity usage would endanger the stability of the grid operation seriously.

From the comprehensive index perspective, the three charging strategies cannot provide satisfied performance under single factor consideration. For instance, the charging at arrival pattern can guarantee the success of traveling but also can produce higher bills; moreover, charging too frequently would be harmful to the battery life. If only the pricing-based charging mode is adopted, the tariff could be least during midnight; however, in addition to a sharp peak would be caused in short period of time, the required electricity could not be guaranteed if the emergency traveling is required.

Here, considering the impact factors involved in the system indicator in equation (1), the three single indices and the compressive index are configured as the objective set, which is denoted by $f_{\text {obj }}$. Then a combined multiobjective charging strategy can be proposed with the developed nonlinear programming model, which is defined as

$$
\begin{aligned}
Y=W_{1} R+W_{2}\left(1.5 \frac{\bar{C}}{C_{\mathrm{TOC}}}\right)+W_{3}\left(1.5 \frac{T_{\text {charge }}}{T_{\text {slack }}}\right)+U(s)+\delta, \\
\text { s.t. }\left\{\begin{array}{c}
\mathrm{SOC}_{\text {min }}, W_{i}, \delta \in[0,1], i=1,2,3, \\
1.0=\sum_{i=1}^{3} W, \\
\max \quad\left\{f_{\text {obj }}=\left\{\mathrm{APR}, R_{\text {save }}, R_{\text {trip }}, Y\right\}\right\},
\end{array}\right.
\end{aligned}
$$

where $W_{i}$ are the coefficients as described in equation (7); $\mathrm{SOC}_{\text {min }}$ directly impacts the required energy supply and the traveling distance of next trip. Given that EV starts charging when $P>0.5$, so $\delta$ is added as the model bias to make the charging decision threshold more reasonable. These parameters are set to be optimized. 


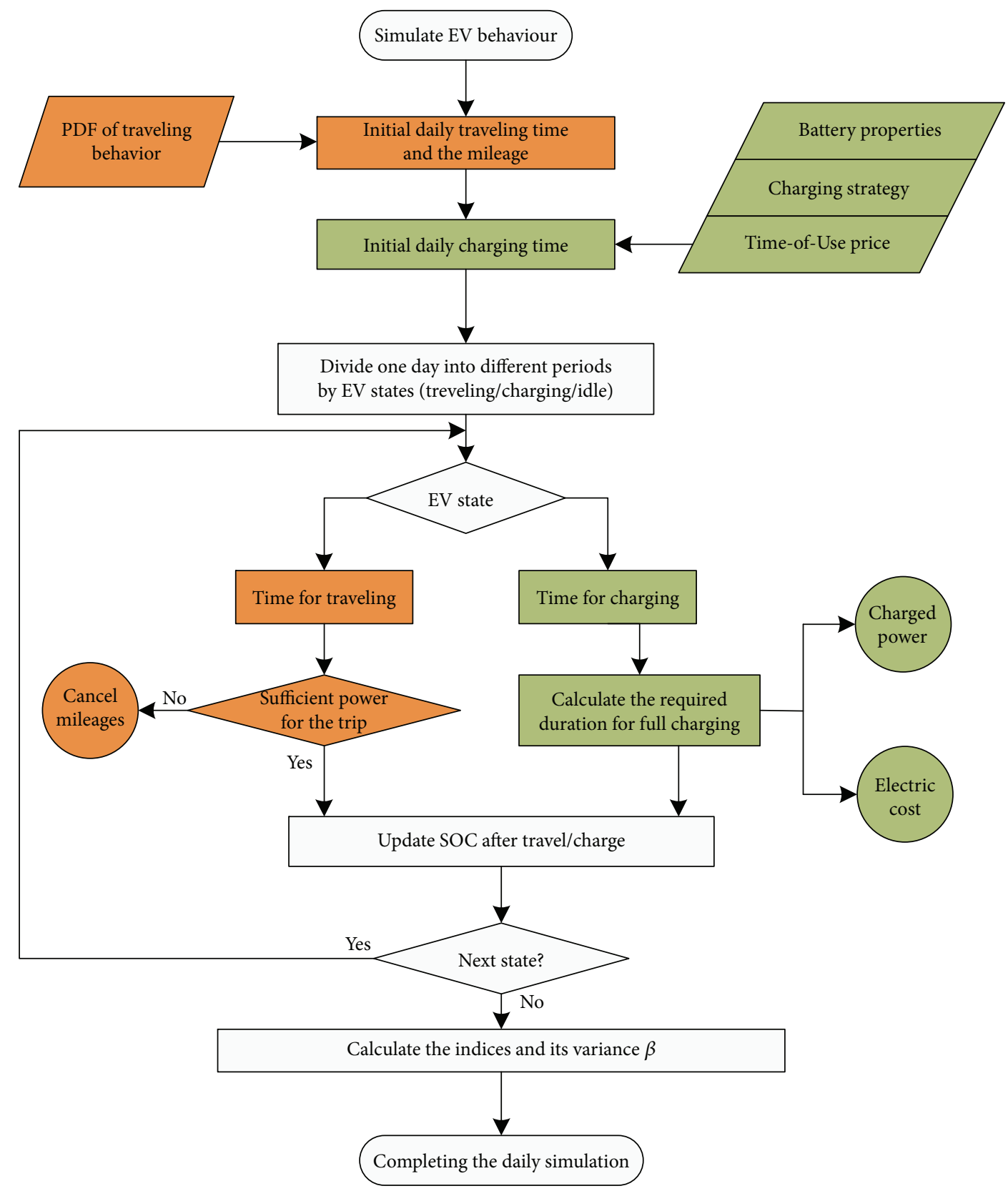

FIgURE 6: The MC simulation of the EV driving and charging behaviour per day.

TABLE 4: The EV daily charging load index prediction in 2020 (unit: MW).

\begin{tabular}{lccccccc}
\hline Charging strategy & Peak value & Average value & Average-peak ratio (APR) & Load rate & Total load & High-peak periods & Low-valley periods \\
\hline Randomness & 245.01 & 53.32 & 0.22 & $1.57 \%$ & 5119.06 & $9: 00 \sim 14: 00$ & $3: 00 \sim 6: 00$ \\
Tariff guiding & 824.64 & 54.70 & 0.07 & $5.29 \%$ & 5251.20 & $23: 00 \sim 2: 00$ & $4: 00 \sim 21: 00$ \\
Parking-charging & 306.92 & 54.87 & 0.18 & $1.97 \%$ & 5267.27 & $20: 00 \sim 23: 00$ & $13: 00 \sim 17: 00$ \\
\hline
\end{tabular}

For the multiobjective problems, previous studies suggest the gradient-based solution [24, 25]. As described in [25], when the objective function is nondifferentiable, gradientbased optimization techniques are not suitable for dynamic programming models with constraints. Moreover, there are many potential local extrema in the charging model, and the gradient descent algorithm can easily lead to pseudoconvergence. Considering that our problem has constraints of equality and inequality, the objective function is not required to be continuous and differentiable in heuristic algorithm; the 


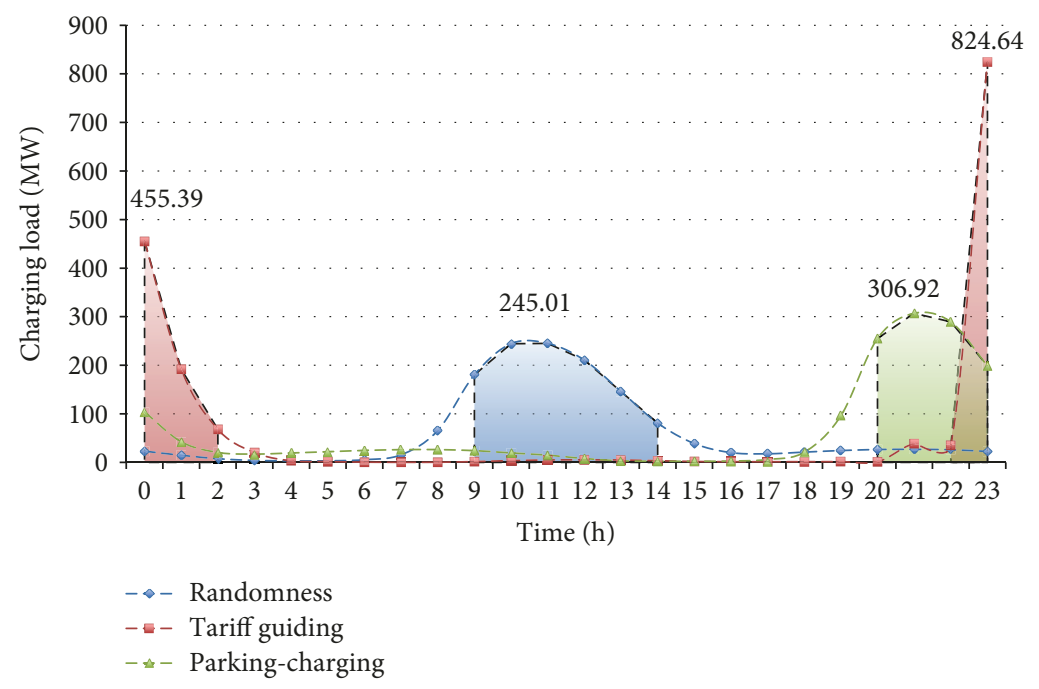

FIGURE 7: The hourly charging loads under 3 different strategies.

1: Var is a variable and its value is at $0.0-1.0$

2: NVar $\leftarrow$ Convert float values $(\operatorname{Var} \times 255)$ to integer values

3: $B$ Var $\leftarrow$ Convert integer values (NVar) to binary values with 8 bits

Algorithm 2: Convert variables to binary.

multiobjective optimization algorithm based on feature selection is proposed to find the global optimal combination of system parameters.

First, the objective function is established as $f_{\text {obj; }}$; three most popular heuristic algorithms, i.e., particle swarm optimization (PSO) [26], genetic algorithm (GA) [27], and simulated annealing (SA) [28] are selected to search the nondominated set. Then the maximized comprehensive index, $\max Y$, can be extracted from the Pareto-front space, so that a unique optimal solution can be obtained via the proposed algorithm.

4.1. Optimal Searching Algorithm under Constraints. According to the constraints expressed in equation (13), a feature selection-based algorithm is proposed to solve the problem from continuous optimization transformed into discrete optimization. First, the involved variables are coded into binary form. Then the feature selection is applied to optimize the feature code of each individual so as to improve the quality of the variables combination under constraints. The related open-source algorithms can be referred in [16], and the detailed steps are described as follows.

Step 1. Preprocess each input variables, $W_{i}, \mathrm{SOC}_{\min }$, and $\delta$ with 8-bit binary coded data, as Algorithm 2

Step 2. Initialize the particles swarm; each is expressed by an 8 -bit $\times 5$ (variables)
Step 3. With the adoption of feature selection algorithms, e.g., BPSO, optimize the feature code of each individual so as to improve the quality of the variable combination

Step 4. Perform the inverse procedure of Step 1 and transform the binary code of each individual into variables set form in order to calculate the individual fitness. The pseudocode (Algorithm 3) are displayed to describe the equation constraint

Step 5. Implement the MC procedure to acquire the fitness, and the converged negative index $\left(1-f_{\text {obj }}\right)$ is regarded as the individual fitness. The related parameters are set to speed up the calculation and guarantee convergence as days $=30$, $\mathrm{EVs}=100$, and variance $=0.005$

Step 6. If the optimality condition is satisfied or the maximum number of iterations has been reached, the procedure stops and the optimal variable combination solution is obtained; otherwise, go to Step 3 to continue the next iteration

Compared to the general constraint-handling methods, such as penalty function [29], the proposed method possesses simplicity and high efficiency by transforming the continuous combination problem into a binary feature optimal problem. It can avoid invalid searching due to a variable out of bound in each iteration. Therefore, binary feature optimization can be applied in multiple intelligent optimal algorithms. 


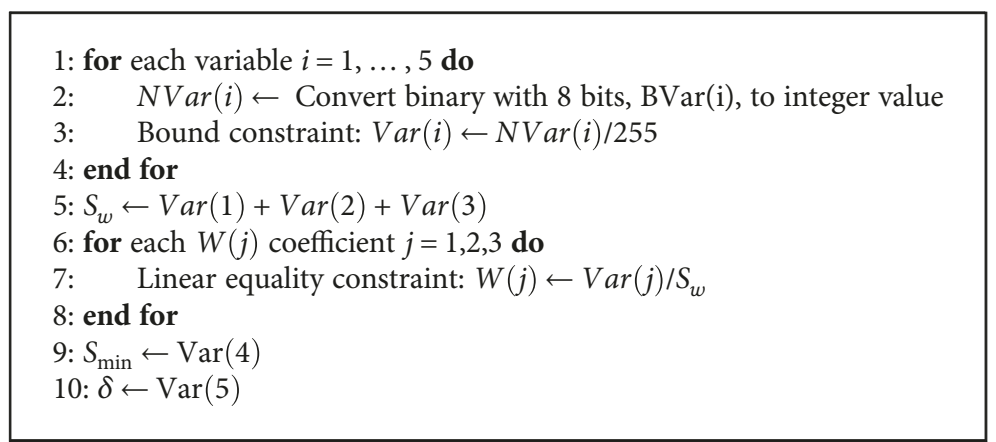

Algorithm 3: Process constraints.

The PSO, GA, and SA are the typical algorithms for the solution of multiobjective problems with constraints. Combined with the basic feature selection method, they are used to solve the objective in equation (13) with multiconstraint. The involved parameter settings are listed in Table 5.

4.2. Experimental Results and Analysis. In order to investigate the access impact of the large-scale EVs to the grid load features, the simulations are kept running for 3 days with two cluster servers, whose hardware are listed in Table 6. The EV charging behaviours are analyzed with multiple charging strategies, and the performance index difference with multiobjective schemes are also discussed.

4.2.1. The Convergence Analysis of the MC Process. The results of the EVs charging in 100 days under different charging strategies via MC simulation are shown in Figure 8 . The variance coefficient of the comprehensive $Y$ value (calculated by equation (13)) can be converged lower than $0.5 \%$, where the reliability is satisfied with the MC simulation requirement.

Based on the traveling model with certain credibility and different charging modes, MC is used to simulate the 240,000 EVs driving in 100 working days. After the convergence of the simulated system is achieved, the daily average charging load curves can be acquired with four different strategies, as shown in Figures 9 and 10(a).

4.2.2. The Performance Comparison of the Charging Strategies. The index comparison of different charging schemes is illustrated in Figure 10. First, under the random strategy, there will be new loads added as a superposition during the peak periods of daytime, and the scheduled trips of EV could be cancelled with higher probability due to insufficient capacity (see Figure 10(c)). Moreover, a sharp peak load could be generated in the midnight with the tariff-guiding strategy. As for the parking-charging strategy, a peak load as well as the highest electricity payment during the evening period could be generated (see Figure 10(b)).

However, the multiobjective charging strategy can achieve a compromise among different indicators (see Figure 9). The essence of the multiobjective is to balance the resource allocation among indicators, and the selection of weights in the formula is rather subjective. Therefore, heuristic algorithm is used to determine the Pareto optimum of variables.
TABle 5: Parameters settings for three heuristic algorithms.

(a)

\begin{tabular}{lccccc}
\hline \multicolumn{7}{c}{ BPSO algorithm } \\
Iterations & Population & Dimension & $\omega$ & $C_{1}$ & $C_{2}$ \\
\hline 200 & 100 & 40 & 0.9 & 1.5 & 1.5 \\
\hline
\end{tabular}

(b)

\begin{tabular}{lcc}
\hline & Genetic algorithm & \\
Operation & Methods & Ratio \\
\hline Selection & To keep certain elite individuals & 0.1 \\
Crossover & Double point & 0.7 \\
Mutation & Uniform & 0.2 \\
\hline
\end{tabular}

(c)

\begin{tabular}{lcccc}
\hline Iteration & Dimension & $\begin{array}{c}\text { SA algorithm } \\
\text { Reanneal } \\
\text { interval }\end{array}$ & $\begin{array}{c}\text { Acceptance } \\
\text { function }\end{array}$ & $\begin{array}{c}\text { Initial } \\
\text { temperature }\end{array}$ \\
\hline 1000 & 40 & 50 & $\begin{array}{c}\text { Boltzmann } \\
\text { probability }\end{array}$ & 100 \\
\hline
\end{tabular}

Table 7 summarizes the indexes of different charging strategies during daily driving. The APR is the highest with random charging mode; the charging tariff is the least with tariff-guiding mode; the highest rate of success traveling can be achieved via parking-charging model; the best comprehensive index is achieved via the multiobjective charging strategy. The results demonstrate that the combination model described in equation (13) and the parameters configuration obtained via SA can guide the charging behaviour effectively and improve the performance indices greatly.

4.2.3. Observations and Discussion. The experimental results shown in Figure 10(a) indicate that the power load will reach 245.01 MW during peak periods in 2020 with $240,000 \mathrm{EVs}$ charging without any guidance in Shenzhen. The power suppliers have to take measures to update the current grid infrastructure and develop the smart grid to reduce the obviously peak-valley difference. Thus, based on the feature 
TABLE 6: The hardware environment of the experiments.

\begin{tabular}{lcc}
\hline Machine & CPU & Memory \\
\hline Cisco, UCSC-C240-M4L & $2 \times$ E5-2609 v4 $(1.7 \mathrm{GHz})$ & $2 \times$ DDR4 $(8 \mathrm{G}, 1866 \mathrm{MHz})$ \\
DELL, PowerEdge R630 & E5-2640 v4 (2.4 GHz) & DDR4 (8 G, 2133 MHz) \\
\hline
\end{tabular}

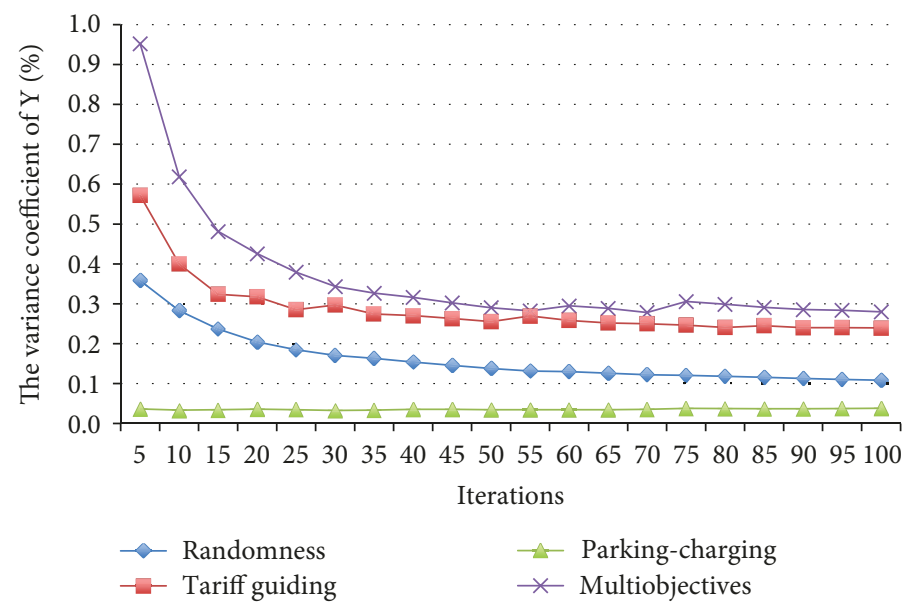

FIgURE 8: MC convergence under 4 different charging strategies.

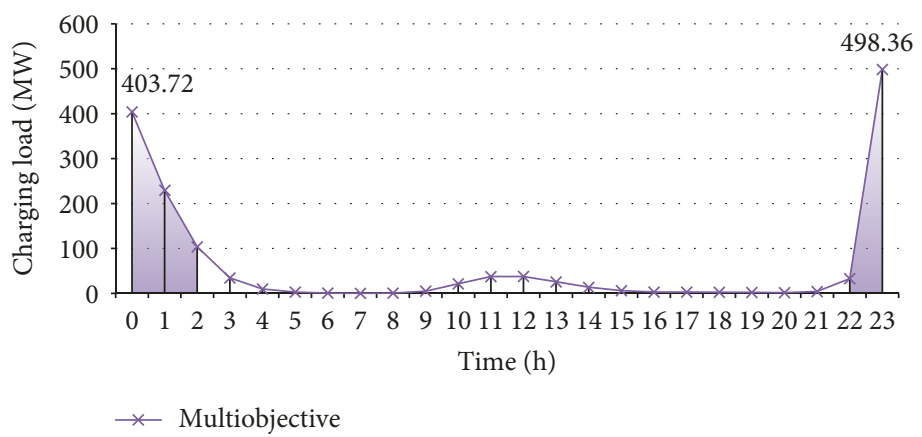

Figure 9: The hourly charging load via the multiobjective strategy.

selection, three optimal searching algorithms considering constraints are proposed and applied for solving multiobjective charging problem. As listed in Table 7, under the multiobjective strategy and its optimized parameters configuration, the APR is increased by $11 \%$, charging cost is saved by $66.2 \%$, and the successful traveling rate is $99.5 \%$. Therefore, the multiobjective combination of charging strategy can provide a maximized comprehensive benefit for the $\mathrm{EV}$ users and power suppliers. From the users perspective, it is suggested that the EV should be charged timely if its SOC is less than $56.9 \%$.

Differentiating from other literatures that concern about the accuracy verification of the load prediction $[30,31]$, this paper designs a universal model to determine the optimal periods for stand-alone charging behaviours, which has no direct communication between the grid and EVs $[11,13]$ or the online adjustment on the electricity price [12]. Referring to the multiobjective strategy, the on-board programmable control system can estimate the optimal periods to charge the EV automatically during EV slack periods set by the EV owners. Not only the traveling plan can be guaranteed but also the electricity bills saving and off-peak energy usage can be achieved simultaneously.

\section{Conclusions}

This paper investigates the EVs large-scale charging behaviours and discusses intelligent charging strategies. First, the probability distribution model of the traveling pattern based on the actual traffic data is established to improve the credibility of the prediction. Considering the benefits for the EV users and power suppliers, the evaluation indices are selected, including load peak value, charging bills, and traveling rate. Under the constraints of the EV battery features and users traveling statistics characteristics, the multiobjective charging strategy has been developed, where the Pareto optimum of 


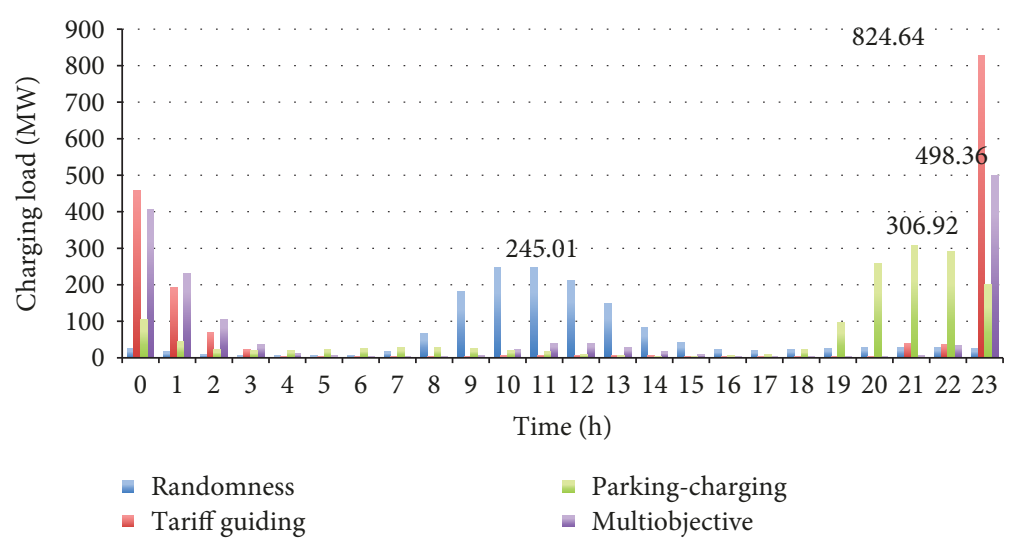

(a) The hourly charging loads from the EVs

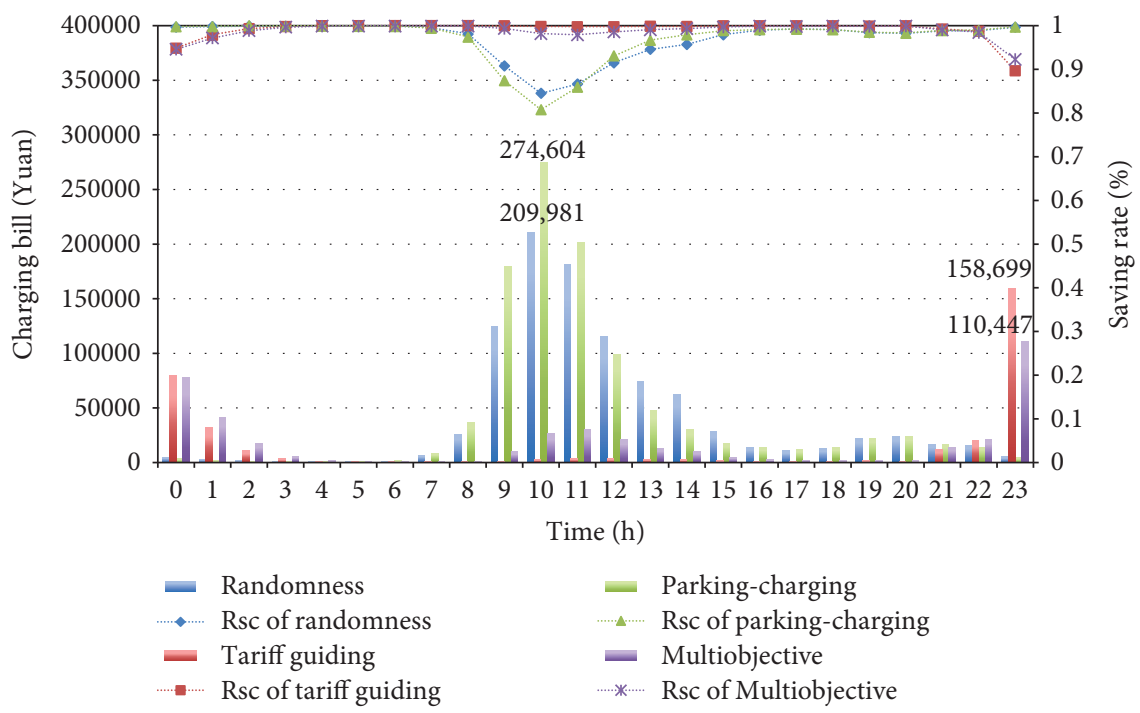

(b) The hourly charging bills (left $\mathrm{Y}$-axis) and the saving rates (right $\mathrm{Y}$-axis)

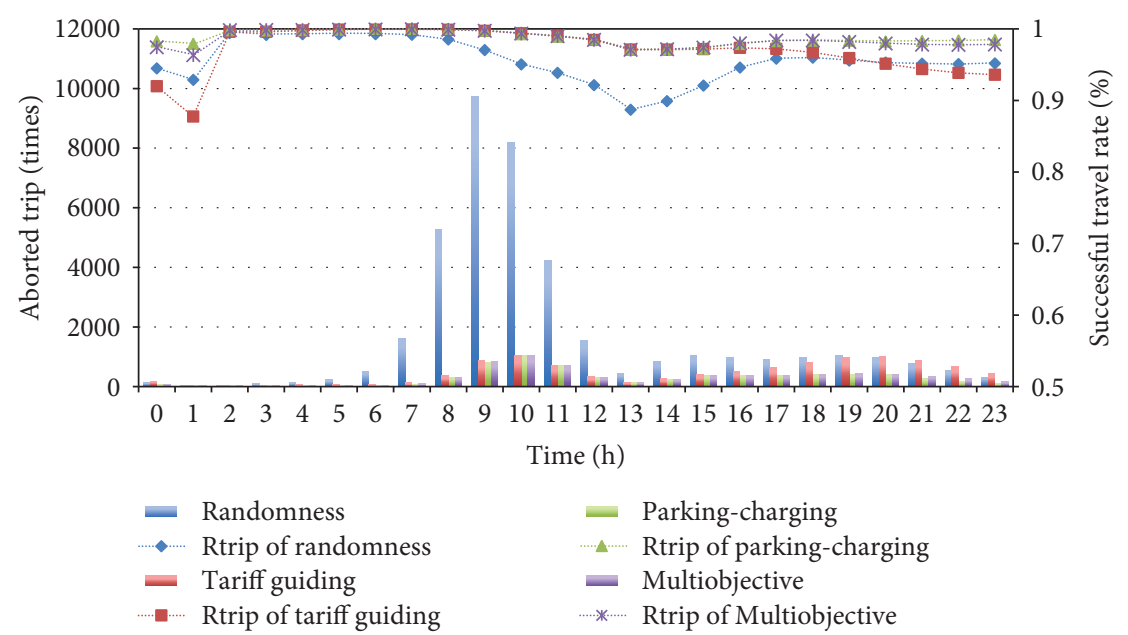

(c) The hourly aborted trips (left $\mathrm{Y}$-axis) and the traveling rates (right $\mathrm{Y}$-axis)

FIGURE 10: Index comparison of the hourly charging under four different charging strategies.

the parameters are determined via a novel constrainthandling method with feature selection. Monte Carlo tool is adopted to simulate the EVs activities, and an open-source system with a universal framework is released to promote the research on access impact of the large-scale EVs to the local grid load.

In summary, the adopted probability distribution statistics, MC simulation, evaluation indices, and EV charging 
TABLE 7: The index comparison of different charging strategies during daily driving.

\begin{tabular}{lcccccc}
\hline Charging strategies & Total load (MW) & APR & Charging bill (Yuan) & Saving rate & Aborted trips (times) & $\begin{array}{c}\text { Traveling rate } \\
\text { Comprehensive } \\
\text { index Y }\end{array}$ \\
\hline Randomness & 5119.06 & 0.218 & 950,703 & 0.193 & 40,098 & 0.972 \\
Tariff guiding & 5251.20 & 0.066 & 335,935 & 0.722 & 10,143 & 0.489 \\
Parking-charging & 5267.17 & 0.179 & $1,014,264$ & 0.163 & 6277 & 0.993 \\
Multiobjective & $\mathbf{5 2 6 2 . 5 7}$ & $\mathbf{0 . 1 1 0}$ & $\mathbf{4 0 8 , 9 4 5}$ & $\mathbf{0 . 6 6 2}$ & $\mathbf{6 6 2 3}$ & $\mathbf{0 . 9 9 5}$ \\
\hline
\end{tabular}

model can provide an alternative for the discussion of largescale EVs development and grid resources assignment. Further research will investigate the MC-based modelling for different EVs (i.e., public transportation) in holidays and the EV connection mode in the real-world grid network. Besides, more work should be focused on the statistical analysis of EVs access to distributed grid nodes, which can give insights on how to realize the proposed methods in the local distribution grids.

\section{Nomenclature}

\section{Battery}

Cap: Battery capacity, $100 \mathrm{Ah}$

Vol: Voltage, $230 \mathrm{~V}$

$E_{c}$ : $\quad$ Charging efficiency, 0.9

$T_{\mathrm{fc}}$ : Full charged duration, $5 \mathrm{~h}$

$W_{d}$ : Energy consumption, $0.175 \mathrm{~kW} / \mathrm{h} / \mathrm{km}$

SOC: State-of-charge $=$ remainder capacity/rated capacity.

Pillar

$W_{c}$ : Demand power from grid, constant power, $15 \mathrm{~kW}$.

User

$F_{d}: \quad$ Traveling frequency (times) per day

$M_{d}$ : The driving mileage of each traveling, unit: $\mathrm{km}$

$T_{d}$ : $\quad$ The duration of each traveling

$D_{t}$ : $\quad$ The departure time of each traveling

$T_{c}$ : $\quad$ Charging periods

$\mathrm{SOC}_{\text {ini }}$ : The SOC at the initial charging moment

$Q_{c}: \quad$ The required charging power, unit: $\mathrm{kW} / \mathrm{h}$ $Q_{c}=\left(1.0-\mathrm{SOC}_{\text {ini }}\right) \times$ Cap $\times$ Vol.

EV scale

$N_{\mathrm{ev}}$ : The amount of EVs.

\section{Evaluation index}

$W_{\text {peak }}: \quad$ Daily charging peak value

$W_{\text {average }}$ Total load/time interval

APR: $\quad W_{\text {average }} / W_{\text {peak }}$

$R_{\text {load }}: \quad$ Load rate $=W_{\text {peak }} /$ grid peak

$C$ : $\quad$ Charging cost $=$ electricity price $\times$ power consumption

$C_{\max }: \quad$ The highest price $\times$ charging quantity

$R_{\text {save }}$ : Saving rate $=1-C / C_{\max }$
$R_{\text {trip }}: \quad$ Actually driving mileage/planned mileage

$Y: \quad$ The comprehensive index; the higher value means higher system performance.

Multiobjective optimization model

$f_{\text {obj }}$ : Three single indices and the comprehensive index

make up the objective set

$W_{i}: \quad$ The coefficients of the factors $(i=1,2,3$, i.e., randomness, price guidance, and slack time)

$\mathrm{SOC}_{\min }$ : The lowest limit of the battery SOC

$\delta$ : The model bias.

\section{Data Availability}

The data used to support the findings of this study are available from the corresponding author upon request.

\section{Conflicts of Interest}

The authors declare that they have no conflicts of interest.

\section{Acknowledgments}

The work was supported by the Shenzhen Science and Technology Innovation Commission Project Grant Ref. JCYJ20160510154736343 and Ref. JCYJ20170307165442023 and Science and Technology Planning Project of Guangdong Province with Ref. 2017B010117009.

\section{References}

[1] D. B. Richardson, "Electric vehicles and the electric grid: a review of modeling approaches, impacts, and renewable energy integration," Renewable and Sustainable Energy Reviews, vol. 19, pp. 247-254, 2013.

[2] S. Eric, "The impact of growing electric vehicle adoption on electric utility grids," Electric Utilities, 2017.

[3] S. Habib, M. Kamran, and U. Rashid, "Impact analysis of vehicle-to-grid technology and charging strategies of electric vehicles on distribution networks-a review," Journal of Power Sources, vol. 277, pp. 205-214, 2015.

[4] Y. Zhou and X. Li, "The state-of-art of the EV charging control strategies," in 2015 34th Chinese Control Conference (CCC), pp. 7916-7921, Hangzhou, China, July 2015.

[5] R. Garcia-Valle and J. P. Lopes, Eds., Electric Vehicle Integration into Modern Power Networks, Springer Science \& Business Media, New York, NY, USA, 2012.

[6] Q. Wu, A. H. Nielsen, J. Ostergaard et al., "Driving pattern analysis for electric vehicle (EV) grid integration study," in 2010 IEEE PES Innovative Smart Grid Technologies 
Conference Europe (ISGT Europe), pp. 1-6, Gothenberg, Sweden, October 2010.

[7] D. Tang and P. Wang, "Dynamic modeling of electric vehicle movable loads based on driving pattern analysis," in 2014 IEEE PES General Meeting | Conference \& Exposition, pp. 1-5, National Harbor, MD, USA, July 2014.

[8] I. K. A. Aswantara, K. S. Ko, and D. K. Sung, "A centralized EV charging scheme based on user satisfaction fairness and cost," in 2013 IEEE Innovative Smart Grid Technologies-Asia (ISGT Asia), pp. 1-4, Bangalore, India, November 2013.

[9] Society of Automotive Engineers and C. B. Toepfer, SAE Electric Vehicle Conductive Charge Coupler, SAE J 1772, REV. MONTH01 (DOC), California Air Resources Board, 2009.

[10] E. Veldman and R. A. Verzijlbergh, "Distribution grid impacts of smart electric vehicle charging from different perspectives," IEEE Transactions on Smart Grid, vol. 6, no. 1, pp. 333342, 2015.

[11] V. Aravinthan and W. Jewell, "Controlled electric vehicle charging for mitigating impacts on distribution assets," IEEE Transactions on Smart Grid, vol. 6, no. 2, pp. 999-1009, 2015.

[12] S. I. Vagropoulos, G. A. Balaskas, and A. G. Bakirtzis, "An investigation of plug-in electric vehicle charging impact on power systems scheduling and energy costs," IEEE Transactions on Power Systems, vol. 32, no. 3, pp. 1902-1912, 2017.

[13] Z. Yu, S. Chen, and L. Tong, "An intelligent energy management system for large-scale charging of electric vehicles," CSEE Journal of Power and Energy Systems, vol. 2, no. 1, pp. 47-53, 2016.

[14] X. Fernandes, J. Rebelo, J. Gouveia, R. Maia, and N. Bustorff Silva, "On-off scheduling schemes for power-constrained electric vehicle charging," 4OR, vol. 15, no. 2, pp. 163-181, 2017.

[15] R. Rao, H. Cai, and M. Xu, "Modeling electric taxis' charging behavior using real-world data," International Journal of Sustainable Transportation, vol. 12, no. 6, pp. 452-460, 2018.

[16] "The open source simulation of the large-scale electric vehicle charging behaviours," https://cn.mathworks.com/ matlabcentral/fileexchange/62816.

[17] H. Wang, X. Zhang, L. Wu et al., "Beijing passenger car travel survey: implications for alternative fuel vehicle deployment," Mitigation and Adaptation Strategies for Global Change, vol. 20, no. 5, pp. 817-835, 2015.

[18] Implementation Plan of Demonstration and Extension of Energy Conservation and New Energy Vehicles in Shenzhen (2009-2012), Report from Shenzhen Development and Reform Commission, Shenzhen, China, 2010, in Chinese.

[19] J. Lei, W. Duan, X. Dong, and Z. Hu, "Plug-in electric vehicles charging load calculating based on Monte Carlo simulation in Shenzhen," in The 14th China Association for Science and Technology Annual Meeting, pp. 1-6, Hebei, China, 2012.

[20] E. B. Iversen, J. M. Morales, and H. Madsen, "Optimal charging of an electric vehicle using a Markov decision process," Applied Energy, vol. 123, pp. 1-12, 2014.

[21] S. Guindon and O. Gascuel, "A simple, fast, and accurate algorithm to estimate large phylogenies by maximum likelihood," Systematic Biology, vol. 52, no. 5, pp. 696-704, 2003.

[22] E. L. Lehmann and J. P. Romano, Testing Statistical Hypotheses, Springer Science \& Business Media, 2006.

[23] Notice on Publication of Tariff and Peak Tariff Tariffs for Residents, Price Bureau of Guangdong, Government Report, 2015, in Chinese.
[24] C. Liu, Z. Gong, K. L. Teo, J. Sun, and L. Caccetta, "Robust multi-objective optimal switching control arising in 1,3-propanediol microbial fed-batch process," Nonlinear Analysis: Hybrid Systems, vol. 25, pp. 1-20, 2017.

[25] C. Liu, Z. Gong, K. L. Teo, and E. Feng, "Multi-objective optimization of nonlinear switched time-delay systems in fed-batch process," Applied Mathematical Modelling, vol. 40, no. 23-24, pp. 10533-10548, 2016.

[26] Y. Zhou, Y. Chen, G. Xu, Q. Zhang, and L. Krundel, "Home energy management with PSO in smart grid," in 2014 IEEE 23rd International Symposium on Industrial Electronics (ISIE), pp. 1666-1670, Istanbul, Turkey, June 2014.

[27] O. Babatunde, L. Armstrong, J. Leng, and D. Diepeveen, “A genetic algorithm-based feature selection," British Journal of Mathematics \& Computer Science, vol. 4, no. 21, pp. 889905, 2014.

[28] K. A. Dowsland and J. M. Thompson, "Simulated annealing," in Handbook of Natural Computing, G. Rozenberg, T. Bäck, and J. N. Kok, Eds., pp. 1623-1655, Springer, Berlin Heidelberg, 2012.

[29] A. K. Yadav, R. Ranjan, U. Mahbub, and M. C. Rotkowitz, "New methods for handling binary constraints," in 2016 54th Annual Allerton Conference on Communication, Control, and Computing (Allerton), pp. 1074-1080, Monticello, IL, USA, September 2016.

[30] R. C. Leou, J. H. Teng, and C. L. Su, "Modelling and verifying the load behaviour of electric vehicle charging stations based on field measurements," IET Generation, Transmission \& Distribution, vol. 9, no. 11, pp. 1112-1119, 2015.

[31] S. Shafiee, M. Fotuhi-Firuzabad, and M. Rastegar, "Investigating the impacts of plug-in hybrid electric vehicles on power distribution systems," IEEE Transactions on Smart Grid, vol. 4, no. 3, pp. 1351-1360, 2013. 


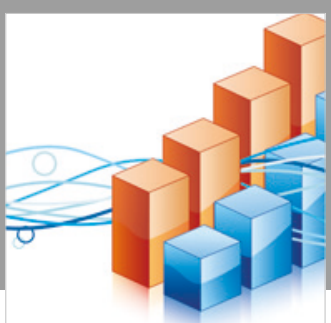

Advances in

Operations Research

\section{-n-m}
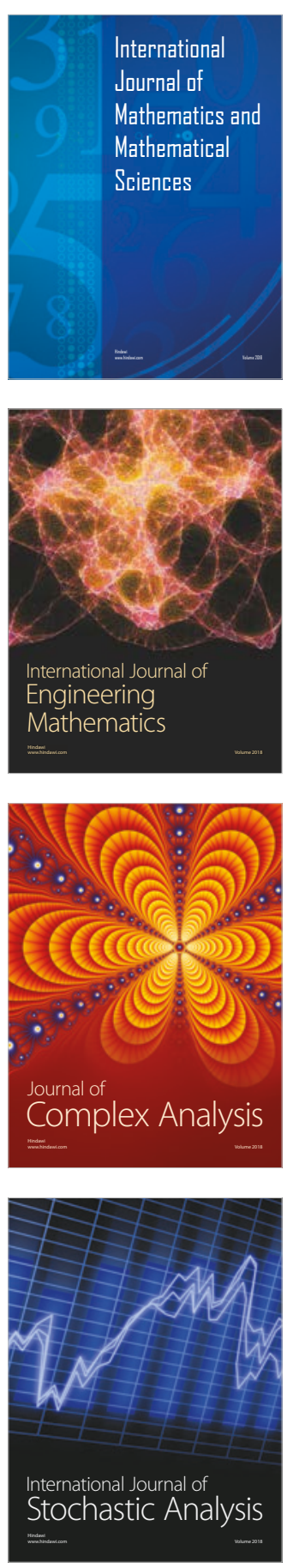
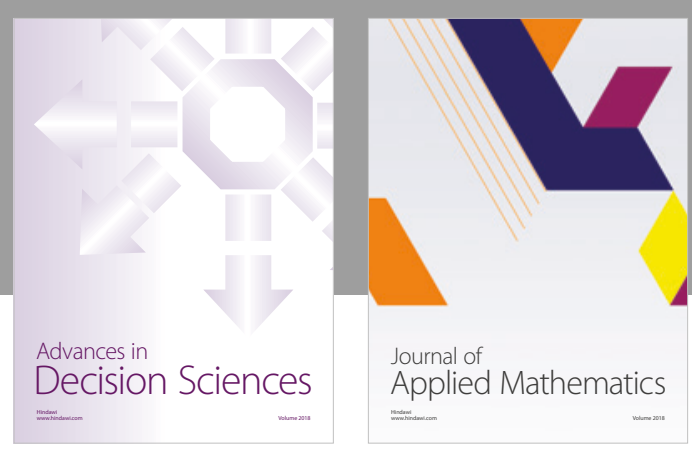

Journal of

Applied Mathematics
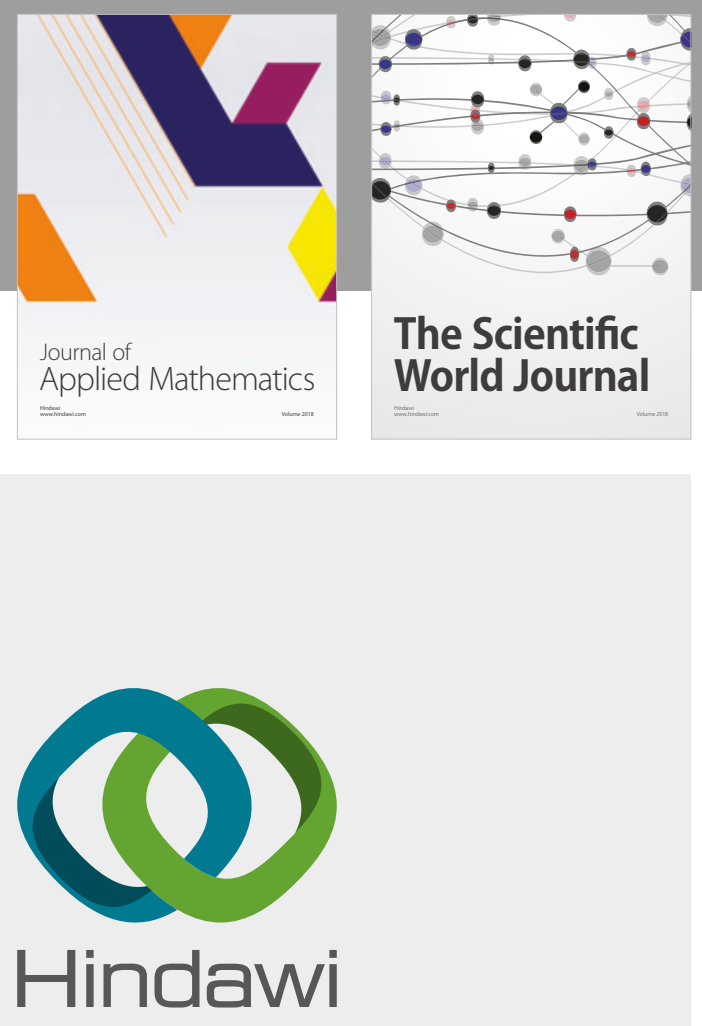

Submit your manuscripts at

www.hindawi.com

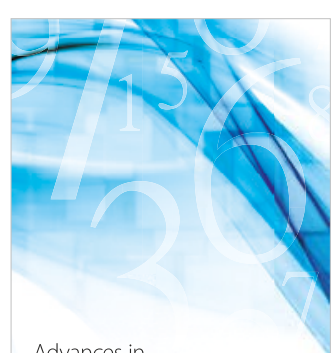

Advances in
Numerical Analysis
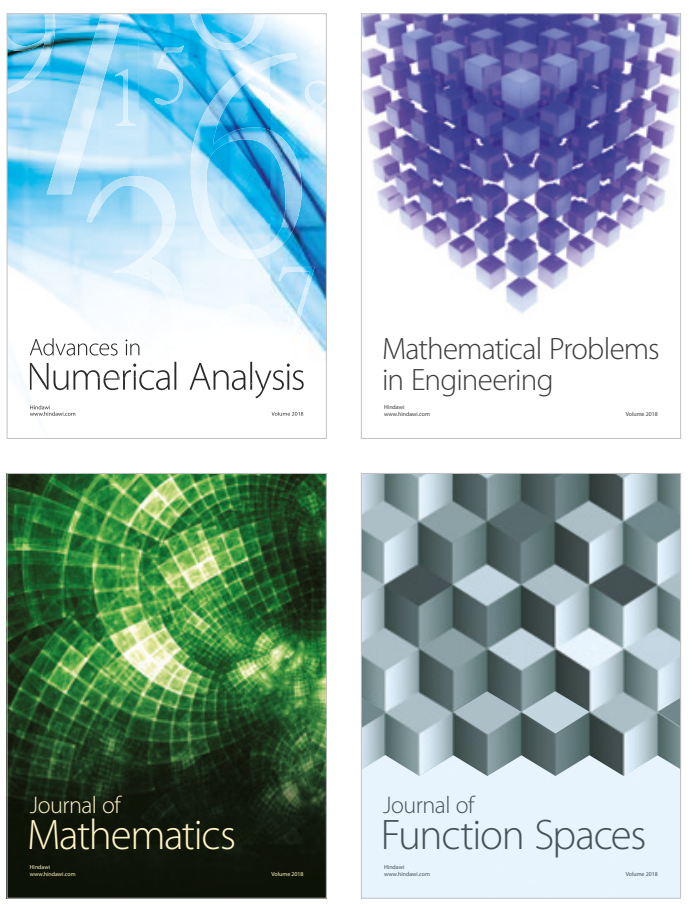

Mathematical Problems in Engineering

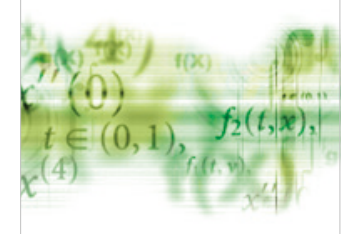

International Journal of

Differential Equations

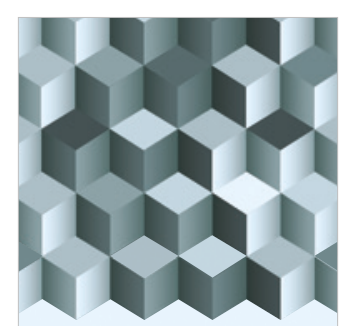

Journal of

Function Spaces
The Scientific

World Journal

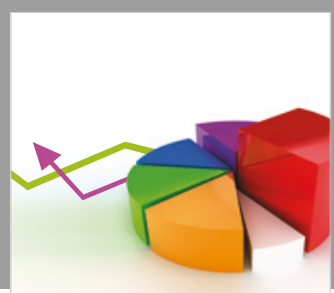

Journal of

Probability and Statistics
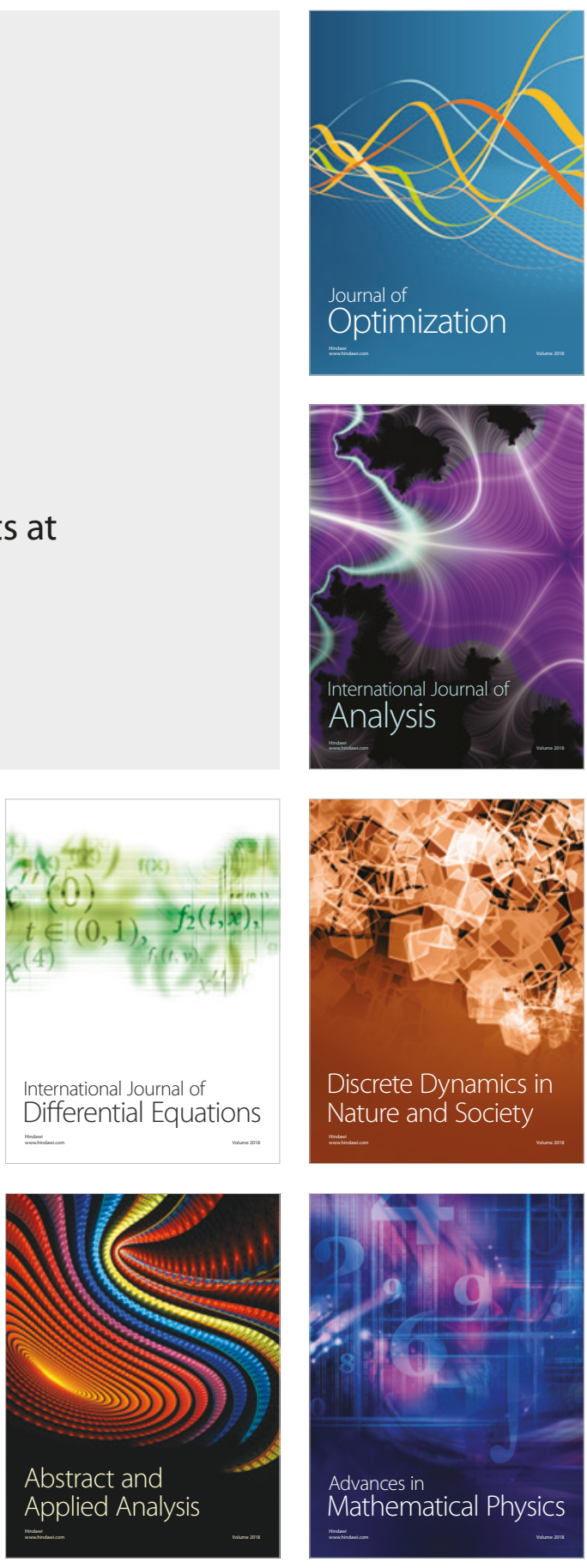